\title{
Proceeding
}

Supplementary Issue: Summer Conferences of Sports Science. $8^{\text {th }}$ International Workshop and Conference of the International Society of Performance Analysis of Sport (ISPAS), 11-1 th of September 2019 (Budapest, Hungary) "Technology meets Practice and Science".

\section{Impact of 24 weeks of strength training coupled to endurance training on endurance performance (HERO Dolomites 2019)}

\author{
LEONARDO CESANELLI $\triangle$, GIORGIA VICI, DALIA CAMILLETTI, ROBERTO CECI, LUCA BELLI, \\ VALERIA POLZONETTI
}

School of Biosciences and Veterinary Medicine, University of Camerino, Camerino, Italy

\begin{abstract}
Despite reports are still equivocal, in recent years different studies reported how strength training could impact positively on endurance performance. The aim of this study was to evaluate the impact of a long-term strength coupled to endurance training protocol in comparison with endurance training protocol on cycling performance. 14 trained amateurs' cyclists (males; 27,03 $\pm 3,75$ years; $177,15 \pm 5,69 \mathrm{~cm} ; 69,67 \pm 8,53 \mathrm{~kg}$; $22,2 \pm 1,67 \mathrm{BMI}$ ) that completed the Hero Dolomites 2018 competition and signed to the 2019 edition (86 $\mathrm{km}, 4500 \mathrm{~m}$ ) participated to the study. 7 of them (treated) followed a 24 weeks strength training coupled to endurance training program to increase performance, the other (untreated) followed their endurance training routines without any specific resistance training protocol. FTP (Functional Threshold Power) test and 1RM (One Repetition Maximum) test (barbell squat and leg press $45^{\circ}$ ) were planned at week 1 , week 8 , week 16 and week 24. 2019 race times were compared to 2018 once. Treated individuals showed increased strength (barbell squat: $+29,7 \pm 7,8 \mathrm{~kg}$; leg press $\left.45^{\circ}:+53,5 \pm 13,1 \mathrm{~kg}\right)$ and FTP $(+23,2 \pm 9,3 \mathrm{~W})$ from week 1 to week 24. Both treated and untreated individuals improved their race time from 2018 to 2019 (treated: $-51,4 \pm 24,6$ m; untreated: $-11,8 \pm 14,4 \mathrm{~m}$ ). Treated individuals improved significantly race time from 2018 compared to untreated individuals $(p<0,05)$. Structured and periodized strength sessions can be incorporated and maintained for the entire duration of a 24 weeks training program impacting positively on endurance performance. Keywords: Cycling; Training; Endurance; Strength.
\end{abstract}

Cite this article as:

Cesanelli, L., Vici, G., Camilletti, D., Ceci, R., Belli, L., \& Polzonetti, V. (2019). Impact of 24 weeks of strength training coupled to endurance training on endurance performance (HERO Dolomites 2019). Journal of Human Sport and Exercise, 14(5proc), S2410-S2418. doi:https://doi.org/10.14198/ihse.2019.14.Proc5.56

Corresponding author. School of Biosciences and Veterinary Medicine, University of Camerino, Camerino, Italy.

E-mail: cesanelli.leonardo@gmail.com

Supplementary Issue: Summer Conferences of Sports Science. $8^{\text {th }}$ International Workshop and Conference of the International Society of Performance Analysis of Sport (ISPAS), 11-13 th of September 2019 (Budapest, Hungary).

JOURNAL OF HUMAN SPORT \& EXERCISE ISSN 1988-5202

(c) Faculty of Education. University of Alicante

doi:10.14198/jhse.2019.14.Proc5.56 


\section{INTRODUCTION}

Strength training (ST) for endurance athletes have received increasing attentions during the last years (Vikmoen et al., 2017; Alcaraz-Ibañez and Rodríguez-Pérez, 2017; Rønnestad and Mujika, 2014; Yamamoto et al., 2010). However, to date, studies and reports about impact of ST on endurance performance are still limited and equivocal (Vikmoen et al., 2017; Yamamoto et al., 2010). Positive effects of ST in endurance athletes have been reported both in running and cycling (Vikmoen et al., 2017). Suggested ST adaptations that may contribute to endurance performance include improvement in economy of movement, delaying of fatigue, improved anaerobic capacity, rate of force development, enhanced maximal speed and positive changes in muscular characteristics (Ronnestad and Mujika, 2014). Anaerobic capacity, maximal speed and motor unit recruitment capacity are examples of possible improvable variables through ST that may have a profound impact in cycling performance (Vikmoen et al., 2017; Ronnestad and Mujika, 2014).

To induce functional adaptations and impact positively on cycling performance, it seems that ST should include heavy loads sessions with velocity during the concentric phase, involving discipline-specific muscle groups and imitating sport-specific movements (Vikmoen et al., 2017; Ronnestad and Mujika, 2014; Beattie et al., 2014; Beattie et al., 2017; Rønnestad et al., 2015; Rønnestad et al., 2017).

Heavy ST can be defined as "all training aiming to increase or maintain muscle or a muscle group's ability to generate maximum force" (Ronnestad and Mujika, 2014). Early phase ST adaptations are mainly related to neuromuscular and connective tissue adaptations (e.g. motor unit recruitment and synchronization), successively followed by muscular adaptations, as a result of progressive increase of training volumes and/or loads (Huges et al., 2017; Yamamoto et al., 2010; Beattie et al., 2014).

Major hypothesis and observations by which ST may contribute positively to endurance cycling performance or in general, endurance, may be relate to: 1) increased maximum strength of type I fibres and consequent postponed time to exhaustion and delayed activation of type II fibres; 2 ) increased proportion of type IIA fibres and reduced proportion of type IIX fibres; 3 ) increased maximum force and/or rate of force development facilitating better blood flow to exercising muscles 4 ) reduction of activated muscle mass to generate the same absolute submaximal power (Ronnestad and Mujika, 2014). However, it is not uncommon, in endurance athletes, to find hesitance to include ST inside season training plans. This can be explained by the possible fear of hypothetical negative effects (e.g. reduced capillary to muscle cross-sectional area ratio) or false myths related to ST on endurance athletes. In this regard, to date, scientific evidence does not support these possible negative effects (Yamamoto et al., 2010; Ronnestad and Mujika, 2014).

Considering studies published in the last two decades (Yamamoto et al., 2010; Beattie et al., 2014; AlcarazIbañez and Rodríguez-Pérez, 2017) our aim was to evaluate the impact of the inclusion of a heavy ST program, maintained for an entire competition preparation, directly on athlete's competition performance. This, comparing data with both: previous results on that competition and with competition performance of athletes training only endurance capacity.

\section{MATERIALS AND METHODS}

\section{Study Design}

To evaluate the impact of ST on endurance performance we compared two different 24 weeks training preparation protocols for an ultra-endurance competition (HERO Dolomites 2019): addition of a strength training protocol to the ongoing endurance training (ST+ET) and endurance training only (ETO). The 
comparisons were made on both, 2019 results ST+ED vs. ETO and between differences of 2018 and 2019 competition results (ST+ED vs. ETO) (figure 1).

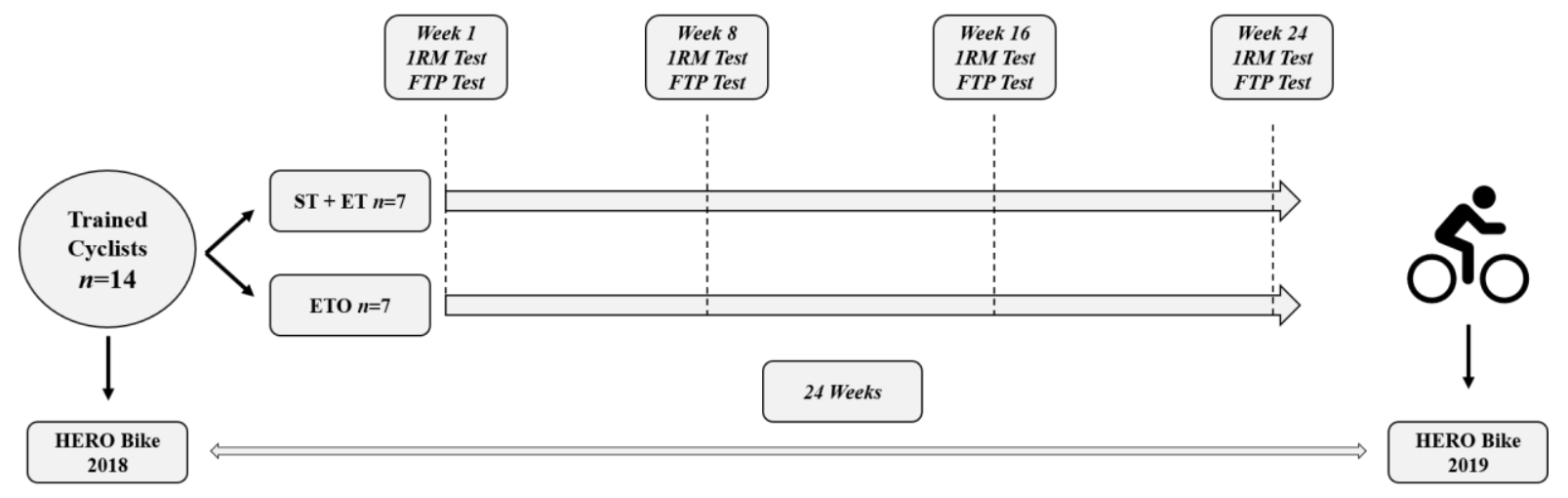

Figure 1. Study design.

\section{Participants}

Inclusion criteria to participate the study were: to be an experienced-well-trained cyclist; to have participated and completed the 2018 edition of the HERO dolomites bike competition; to have not performed in the last six months any kind of strength training or similar resistance training protocols. Fourteen amateur male offroad well-trained cyclists exceeded the inclusion criteria and voluntary accepted to participate the study after signing informed consent and being informed of testing procedures according to the Declaration of Helsinki. Characteristics of the subjects are resumed in table 1 expressed as mean \pm standard deviation.

Table 1. Study participants characteristics.

\begin{tabular}{cccccc}
\hline Group & $\boldsymbol{n}$ & Age (years) & Body Mass $(\mathbf{k g})$ & Height $(\mathbf{c m})$ & BMI $\left(\mathbf{k g} / \mathbf{m}^{2}\right)$ \\
\hline ST+ET & 7 & $26.8 \pm 4.37$ & $66.68 \pm 7.4$ & $175,0 \pm 3.7$ & $21.7 \pm 1.7$ \\
\hline ETO & 7 & $27.2 \pm 3.6$ & $72.6 \pm 9.0$ & $179.3 \pm 6.7$ & $22.5 \pm 1.6$ \\
\hline
\end{tabular}

Values are expressed as mean $\pm S D ; B M I=$ Body Mass Index

\section{Training protocols}

Starting from the basis of what athletes are already doing endurance training guidelines were structured considering FTP tests performed at T1 (week 1). Considering the characteristics of the competition main focus was firstly on endurance capacity (e.g. steady state medium intensity long distance trainings Z2-Z3) and progressively shifted on lactate threshold and neuromuscular capacity (e.g. steady state high intensity intervals or maximal efforts short intervals Z4-Z7). Endurance trainings were performed 3-4 times per week. Heavy strength training structured for ST+ED group consisted on 2 session per week of one hour. Were selected targeting for muscle involved in cycling including exercises mimicking pedalling gesture. Exercises were performed considering a $90^{\circ}$ knee flexion to almost full extension range of motion. Execution was provided explosive concentric phases with slowed eccentric phases, times under tension in general were 1.1.3.1 (figure 2). Sets were structured as classic sets and cluster sets (Tufano et al., 2016). The performed exercises were: full back barbell squat, leg press $45^{\circ}$, horizontal leg press monopodalic, cable kickbacks, leg curl horizontal, seated leg curl monopodalic cable knee rises, core stability exercises. Sets per exercise depended on kind of exercise (from 3 to 5 sets) and repetition depending on load (@1RM). Generally, one or two warm up sets were planned in fundamental exercises (e.g. barbell squat). At least 1 strength session 
per week was supervised. Before starting with the effective training protocol, a first week of familiarization with the exercises was planned. Training intensity (load) referred to $1 \mathrm{RM}$ progressively increase during the weeks. Training intensity was periodized considering 3 mesocycles of 8 weeks with week 1 , week 8 , week 16 and week 24 as deloading and the other with a progressive increase in intensity (load) (figure 2). Cyclists were allowed to have assistance if needed during the execution of heavier load sets. Loads were progressively adjusted considering strength increases and/or daily sensations.
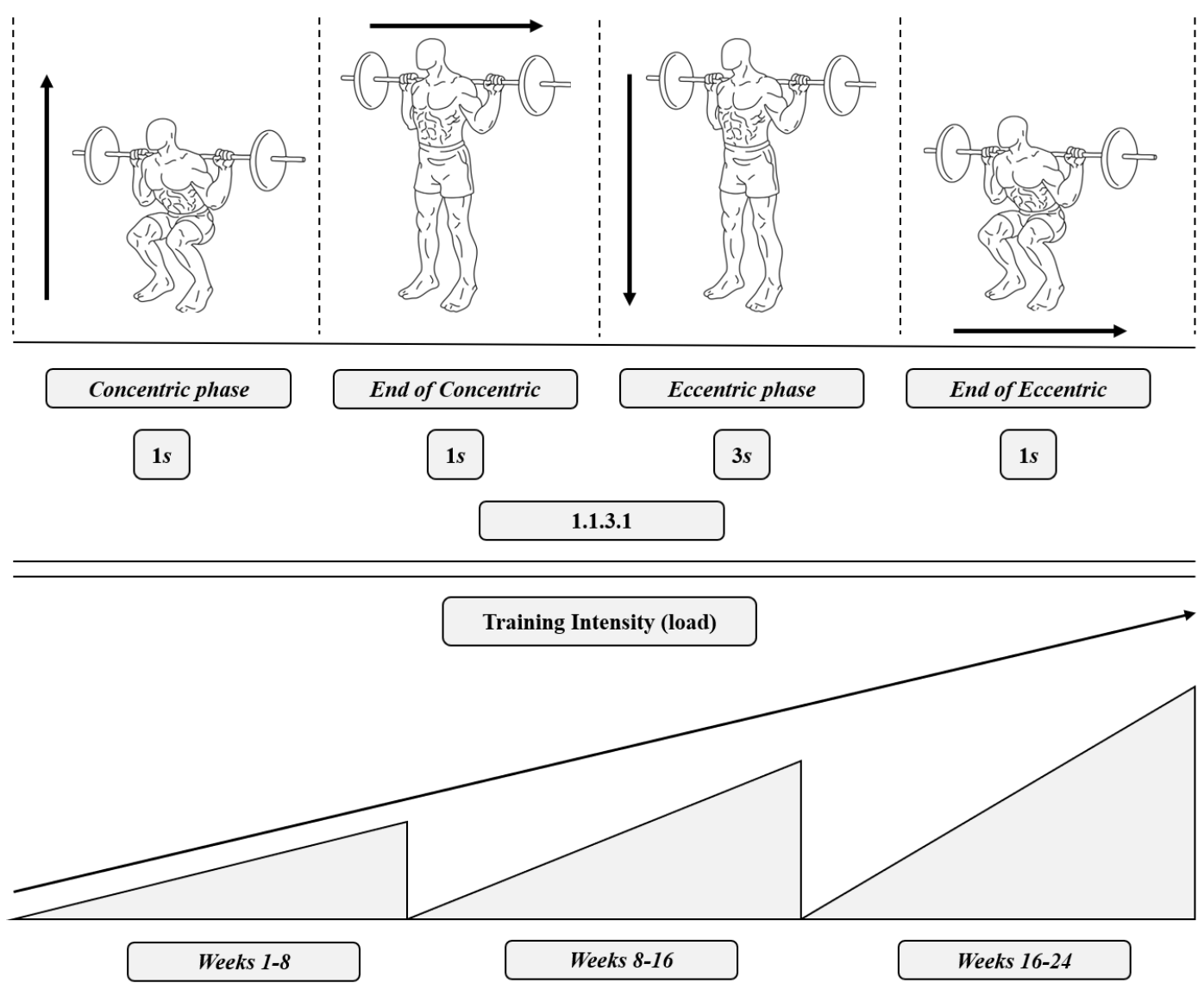

Figure 2. Strength training structure and characteristics.

\section{Physical performance tests}

Physical performance tests were planned at week 1 (T1), week 8 (T2), week 16 (T3) and week 24 (T4). Power output (W) was measured through crankset power meters of the cyclists, heart rate (bpm) and training data were acquired through cyclists cyclocomputers. Functional Threshold Power (FTP) 1-hour test was performed standardizing route, weather conditions and time of the day (Allen et al., 2019). All the tests were performed after a "recovery" (active recovery training session) day. One Repetition Maximum test (1RM) was performed in order to quantify strength. Tests were performed on barbell squat and leg press. Tests started with a specific warm-up of 3-4 sets starting from light loads $( \pm 45-50 \% 1 \mathrm{RM})$ and after that progressively increasing load till reaching approximately the 1RM (maximum amount of weight that a person can lift for a given exercise) (Kraemer et al., 1995). 


\section{Statistical Analysis}

All data in text, tables, figures are represented as mean \pm standard deviation. Differences between groups (race times and FTP comparisons) and within group (1RM test pre and post training protocol completion) paired Student's $t$-test were performed. All analyses resulting in $p \leq 0.05$ were considered statistically significant.

All statistical analyses were carried out using GraphPad Prism version 7.0 (GraphPad Software, San Diego, CA, USA).

\section{RESULTS}

All the athletes completed successfully the training programs and the HERO Bike 2019 competition.

\section{Competition performance}

Both, ST+ET group and ETO group improved their race time from 2018 to 2019. ST+ET group improved significantly race times $(p=0.0015)$ while ETO group improved race times but without statistical significance $(p=0.0723$ ) (figure 3). Comparison between 2018 and 2019 race times resulted in significant differences between groups $(p=0.032)$ with a delta $(\Delta)$ race time of $-51.42 \pm 24.67 \mathrm{~m}$ for ST+ET group and $-11.85 \pm$ $14.04 \mathrm{~m}$ for ETO group (figure 4).
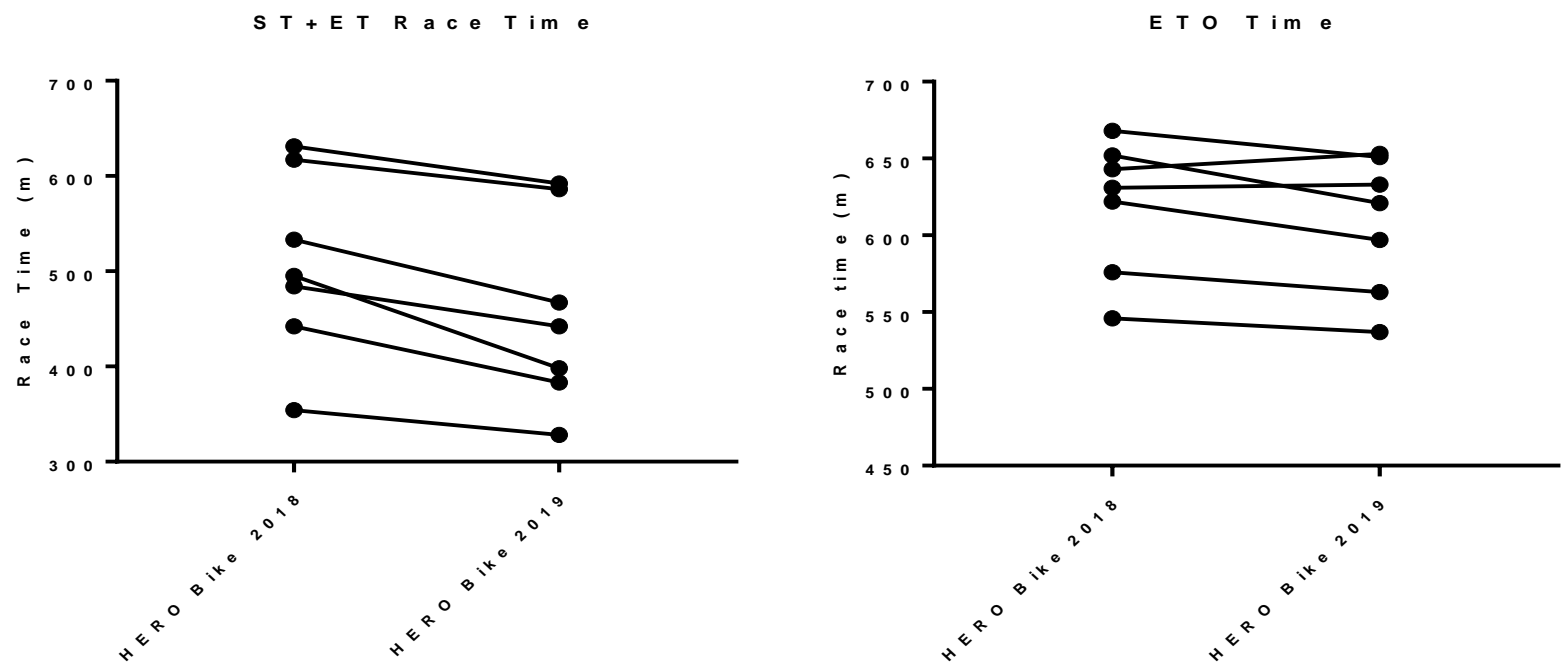

Figure 3. Competition performance results ST+ET and ETO. 


\section{$\Delta$ Race Time ST+ET vs ETO}

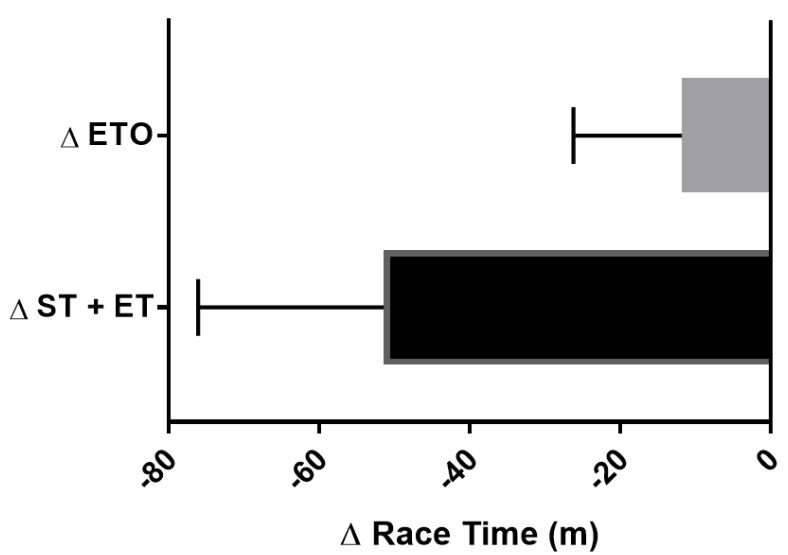

Figure 4. Race time comparison between ST+ET group and ETO group.

\section{Maximal strength}

All ST+ET group athletes increased maximal strength (1RM test) significantly from week 1 to week 24 of training protocol and competition preparation (figure 5). Barbell full squat 1RM increased $29.71 \pm 7.86 \mathrm{~kg} \mathrm{(p}$ $<0.0001)$ and leg press $45^{\circ} 1 \mathrm{RM}$ increased $53.50 \pm 13.16 \mathrm{~kg}(p<0.0001)$.

B a rb e II S q u a $t$ 1 R M

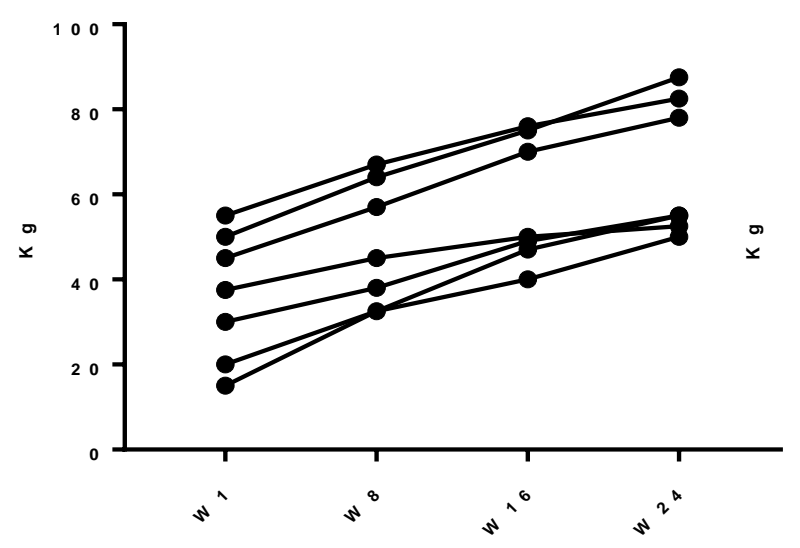

L e g P ress $1 \mathrm{R} M$

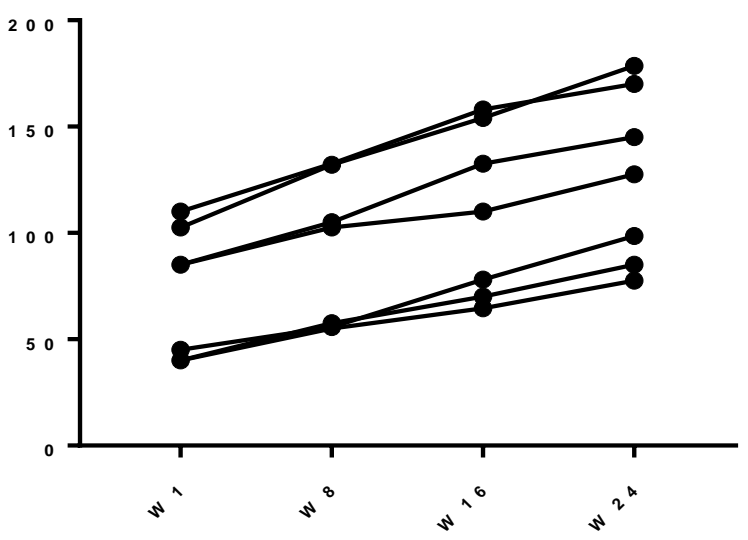

Figure 5. Strength improvements from week 1 to week 24 (1RM test).

\section{Functional Threshold Power}

FTP increased significantly from week 1 to week 24 for both groups. ST+ET group increased FTP $23.28 \pm$ $9.34 \mathrm{~W}(p=0.0006)$ and ETO group improved FTP $22.42 \pm 4.75 \mathrm{~W}(p<0.0001)$ (figure 6). Comparison of FTP improvements of ST+ET and ETO group ( $\triangle$ FTP) from week 1 to week 24 resulted not statistically significant $(p=0.83)$ (figure 7). 

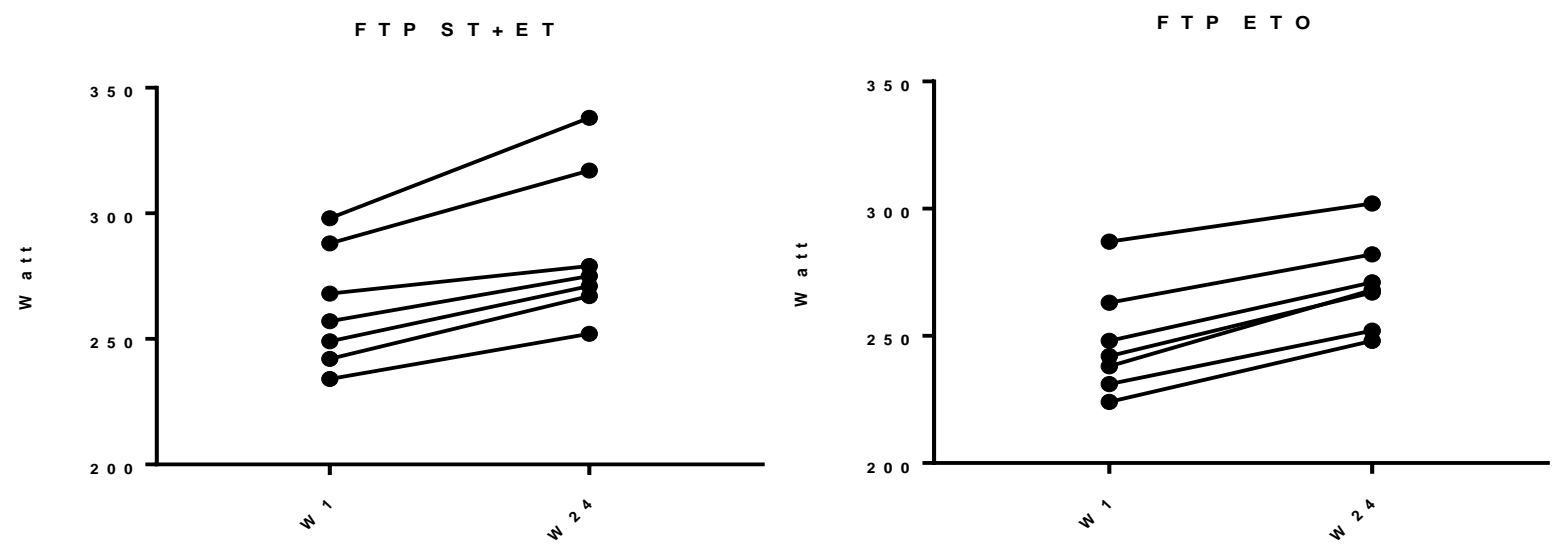

Figure 6. FTP improvements from week 1 to week 24 (ST + ET and ETO).

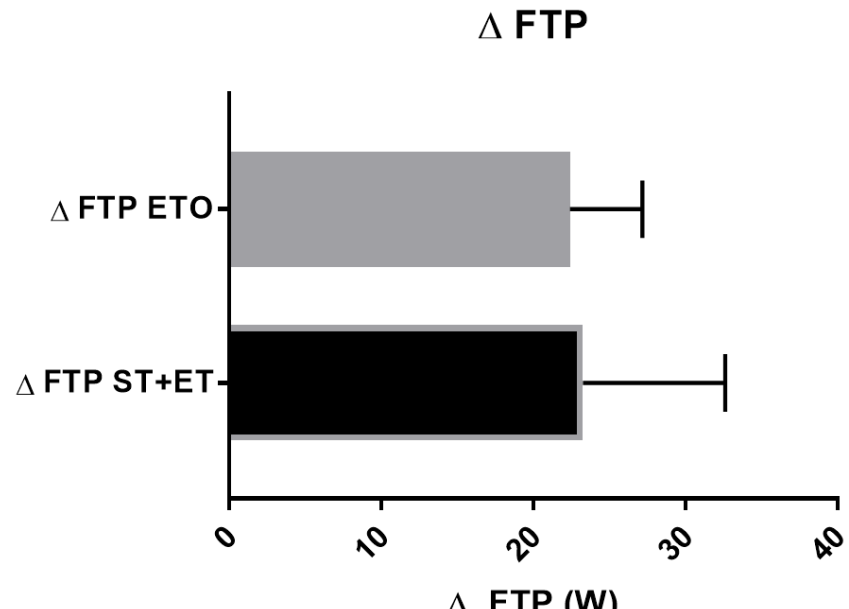

Figure 7. Comparison of FTP at the end of the 24 weeks training program between ST+ET group and ETO group.

\section{DISCUSSION}

Strength training incorporation and in particular its long-term maintenance during the entire training preparation of endurance/ultra-endurance athletes is not common. However, scientific literature has, to date, denied the possibility of incurring in negative 0 detrimental effects and seems to support some positive impacts of strength training on endurance performance (Yamamoto et al., 2010; Beattie et al., 2014; AlcarazIbañez and Rodríguez-Pérez, 2017).

In the present study we analysed the effects of incorporation of strength training sessions in a long-term cycling preparation for an endurance competition. This is the first study that we know on strength training impact on endurance performance in which performance was evaluated analysing the impact directly on competition results. 
Strength training coupled to endurance training impacted positively on athlete's competition performance and produced more marked improvements than the endurance training only group. Improving the FTP is one of the parameters/markers to determine cyclists' physical condition (Allen et al., 2019). However, our data suggest how improving maximum strength as well as FTP seem to be a combination capable of producing more significant improvements on race times.

It is interesting how improvements in terms of FTP between the two groups were similar but how parallelly competition race time compared to the previous year's edition significantly different. Certainly, this aspect could be the subject of further investigation.

\section{CONCLUSIONS}

Structured and periodized strength sessions can be incorporated and maintained for the entire duration of a 24 weeks training program impacting positively on endurance performance.

Further studies or study implementations will be needed to determine the impact of strength training and the dynamics with which it can influence positively endurance performance.

\section{ACKNOWLEDGMENTS}

We would like to thank all the athletes who participated the study. No sources of funding were used in the preparation of this manuscript.

\section{REFERENCES}

Alcaraz-lbañez, M., \& Rodríguez-Pérez, M. (2018). Effects of resistance training on performance in previously trained endurance runners: A systematic review. J. Sports Sci., 36(6), 613-629. https://doi.org/10.1080/02640414.2017.1326618

Allen, H., Coggan, A. R., \& McGregor, S. (2019). Training and racing with a power meter. VeloPress.

Beattie, K., Carson, B. P., Lyons, M., \& Kenny, I. C. (2017). The effect of maximal-and explosive-strength training on performance indicators in cyclists. Int J Sports Physiol Perform, 12(4), 470-480. https://doi.org/10.1123/ijspp.2016-0015

Beattie, K., Kenny, I. C., Lyons, M., \& Carson, B. P. (2014). The effect of strength training on performance in endurance athletes. Sports Med, 44(6), 845-865. https://doi.org/10.1007/s40279-014-0157-y

Hughes, D. C., Ellefsen, S., \& Baar, K. (2017). Adaptations to endurance and strength training. Cold Spring Harb Perspect Med, a029769. https://doi.org/10.1101/cshperspect.a029769

Kraemer, W. J., Fry, A. C., Ratamess, N., \& French, D. (1995). Strength testing: development and evaluation of methodology. Physiological assessment of human fitness, 2, 119-150.

Rønnestad, B. R., \& Mujika, I. (2014). Optimizing strength training for running and cycling endurance performance: A review. Scand J Med Sci Sports, 24(4), 603-612. https://doi.org/10.1111/sms.12104

Rønnestad, B. R., Hansen, J., \& Nygaard, H. (2017). 10 weeks of heavy strength training improves performance-related measurements in elite cyclists. J. Sports Sci., 35(14), 1435-1441. https://doi.org/10.1080/02640414.2016.1215499

Rønnestad, B. R., Hansen, J., Hollan, I., \& Ellefsen, S. (2015). Strength training improves performance and pedaling characteristics in elite cyclists. Scand J Med Sci Sports, 25(1), e89-e98. https://doi.org/10.1111/sms.12257 
Tufano, J. J., Brown, L. E., \& Haff, G. G. (2017). Theoretical and practical aspects of different cluster set structures: a systematic review. J Strength Cond Res, 31(3), 848-867. https://doi.org/10.1519//sc.0000000000001581

Vikmoen, O., Ellefsen, S., Trøen, Ø., Hollan, I., Hanestadhaugen, M., Raastad, T., \& Rønnestad, B. R. (2016). Strength training improves cycling performance, fractional utilization of VO2max and cycling economy in female cyclists. Scand J Med Sci Sports, 26(4), 384-396. https://doi.org/10.1111/sms. 12468

Vikmoen, O., Rønnestad, B. R., Ellefsen, S., \& Raastad, T. (2017). Heavy strength training improves running and cycling performance following prolonged submaximal work in well-trained female athletes. Physiol Rep, 5(5). https://doi.org/10.14814/phy2.13149

Yamamoto, L. M., Klau, J. F., Casa, D. J., Kraemer, W. J., Armstrong, L. E., \& Maresh, C. M. (2010). The effects of resistance training on road cycling performance among highly trained cyclists: a systematic review. J Strength Cond Res, 24(2), 560-566. https://doi.org/10.1519/jsc.0b013e3181c86583

\section{@) $\odot \Theta \Theta$}

This work is licensed under a Attribution-NonCommercial-NoDerivatives 4.0 International (CC BY-NC-ND 4.0). 